\title{
Quenched Scaling Study of Charm and Bottom Systems with a Relativistic Heavy Quark Action
}

\section{CP-PACS Collaborations:}

Y. Kuramashi ${ }^{* a, b} \dagger$, S. Aoki ${ }^{b, c}$, O. Bär ${ }^{b}$, K.-I. Ishikawa $^{g}$, T. Ishikawa ${ }^{a}$, N. Ishizuka ${ }^{a, b}$, Y. Iwasaki ${ }^{b}$, K. Kanaya ${ }^{b}$, T. Kaneko ${ }^{e, f}$, M. Okawa $^{g}$, Y. Taniguchi $^{a, b}$, N. Tsutsui ${ }^{e}$, A. Ukawa ${ }^{a, b}$, N. Yamada ${ }^{e}$ and T. Yoshié Ya,b $^{a, b}$

${ }^{a}$ Center for Computational Sciences, University of Tsukuba, Tsukuba, Ibaraki 305-8577, Japan

${ }^{b}$ Graduate School of Pure and Applied Sciences, University of Tsukuba, Tsukuba, Ibaraki 305-8571, Japan

${ }^{c}$ Riken BNL Research Center, Brookhaven National Laboratory, Upton, New York 11973, USA

${ }^{d}$ Institute for Cosmic Ray Research, University of Tokyo, Kashiwa 277-8582, Japan

${ }^{e}$ High Energy Accelerator Research Organization (KEK), Tsukuba 305-0801, Japan

${ }^{f}$ School of High Energy Accelerator Science, The Graduate University for Advanced Studies

(Sokendai), Tsukuba 305-0801, Japan

${ }^{g}$ Department of Physics, Hiroshima University, Higashi-Hiroshima, Hiroshima 739-8526, Japan

\begin{abstract}
We present a detailed scaling study of the charm and bottom systems using our relativistic heavy quark action and the Iwasaki gauge action in quenched QCD. We investigate two cases: (i) all the four parameters $v, r_{s}, c_{B}, c_{E}$ in the heavy quark action are determined up to one-loop level, and (ii) the parameter $v$ is nonperturbatively determined from the dispersion relation of the quarkonium with $r_{s}, c_{B}, c_{E}$ left at the one-loop level. We measure the charmonium and bottomonium spectra including both spin-independent and spin-dependent splittings, heavy-light pseudoscalar decay constants and charm and bottom quark masses. The results for the bottom system show good scaling behavior compared to those with NRQCD. This feature is further improved, once the nonperturbative $v$ is employed.
\end{abstract}

XXIIIrd International Symposium on Lattice Field Theory

25-30 July 2005

Trinity College, Dublin, Ireland

* Speaker.

${ }^{\dagger}$ E-mail: kuramasi@ het.ph.tsukuba.ac.jp 


\section{Introduction}

Lattice QCD should be an ideal tool to provide quantitative predictions for heavy quark physics from first principles. However, there is an obstacle which prevents us from achieving this goal: large $m_{Q} a$ corrections at lattice spacings accessible with current computational resources.

Recently, we have proposed a new relativistic approach to control the $m_{Q} a$ corrections from the view point of the on-shell $O(a)$ improvement program[1]. The relativistic heavy quark (RHQ) action is given by

$$
\begin{aligned}
S_{q}= & \sum_{x}\left[m_{0} \bar{q}(x) q(x)+\bar{q}(x) \gamma_{0} D_{0} q(x)+v \sum_{i} \bar{q}(x) \gamma_{i} D_{i} q(x)-\frac{r_{t} a}{2} \bar{q}(x) D_{0}^{2} q(x)-\frac{r_{s} a}{2} \sum_{i} \bar{q}(x) D_{i}^{2} q(x)\right. \\
& \left.-\frac{i g a}{2} c_{E} \sum_{i} \bar{q}(x) \sigma_{0 i} F_{0 i} q(x)-\frac{i g a}{4} c_{B} \sum_{i, j} \bar{q}(x) \sigma_{i j} F_{i j} q(x)\right]
\end{aligned}
$$

where we are allowed to choose $r_{t}=1$, while the other four parameters $v, r_{s}, c_{E}, c_{B}$ should be adjusted as analytic functions of $m_{Q} a$ and the gauge coupling constant $g$ from relativistic invariance to $O(a)$ for arbitrary magnitude of $m_{Q}$. In Ref.[2] we have determined the four improvement parameters up to one-loop level for various improved gauge actions employing the on-shell quark-quark scattering amplitude. Furthermore, we have carried out a perturbative determination of mass dependent renormalization factors and $O(a)$ improvement coefficients for the vector and axial vector currents[3, 4].

In this report we make a quenched scaling study of the charm and bottom systems with the RHQ action and the Iwasaki gauge action[5]. We test two choices for the parameters in the RHQ action. In one choice designated as RHQ(PT), $v, r_{s}, c_{E}, c_{B}$ are perturbatively determined. In the other choice called RHQ(NP), $v$ is nonperturbatively determined, while the others remain perturbative. We have investigated various physical quantities: the charmonium and bottomonium spectra, heavy-light pseudoscalar decay constants and heavy quark masses. Our results are compared to those with the clover quark action on isotropic and anisotropic lattices for the charm system, while for the bottom system we employ the NRQCD results as a benchmark.

\section{Simulation details}

In Table 1 we summarize parameters of our quenched simulations with the Iwasaki gauge action. The lattice spacing $a$ at each $\beta$ is from a fit of $a$ as a function of $\beta$ using $r_{0}=0.5 \mathrm{fm}[6]$. The physical spatial size is chosen to be $L a=1.8 \mathrm{fm}$. The same gauge configurations are used for

Table 1: Simulation parameters.

\begin{tabular}{llll}
\hline$L^{3} \times T$ & $\beta$ & $a[\mathrm{fm}]$ & $\#$ conf $(v$ tuning $)$ \\
\hline $16^{3} \times 40$ & 2.5120 & 0.11250 & $550(150)$ \\
$20^{3} \times 48$ & 2.6606 & 0.09000 & $480(160)$ \\
$24^{3} \times 48$ & 2.7863 & 0.07500 & $450(180)$ \\
$32^{3} \times 64$ & 2.9939 & 0.05625 & 420 \\
\hline
\end{tabular}



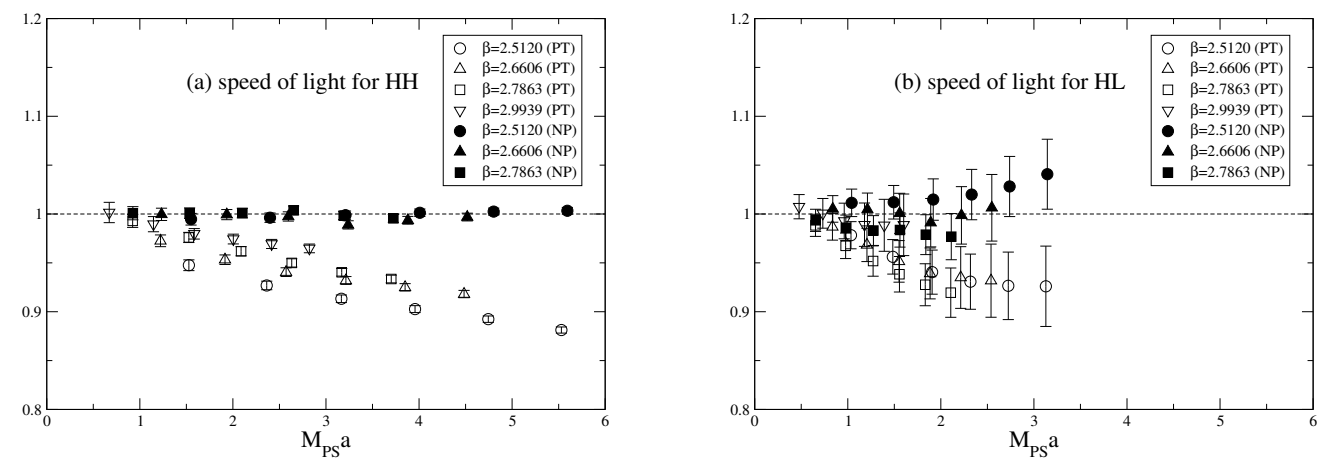

Figure 1: Speed of light for (a) heavy-heavy and (b) heavy-light pseudoscalar mesons.

the comparison of $\mathrm{RHQ}(\mathrm{PT})$ and $\mathrm{RHQ}(\mathrm{NP})$. The simulation with $\mathrm{RHQ}(\mathrm{NP})$ at $\beta=2.9939$ is now under way.

For the heavy quark parameters in the RHQ action (1.1), we impose $r_{t}=1, r_{s}$ is calculated at one-loop level, and $v$ is either perturbatively or nonperturbatively determined. For $c_{B}$ and $c_{E}$ we adopt the following procedure to incorporate nonperturbative contribution at $m_{Q}=0$ :

$$
c_{B / E}=\left\{c_{B / E}^{\mathrm{PT}}\left(m_{Q} a\right)-c_{B / E}^{\mathrm{PT}}(0)\right\}+c_{\mathrm{SW}}^{\mathrm{NP}} .
$$

At each $\beta$ we choose six values of hopping parameters ranging from charm to bottom quark masses. For the light quarks we use the nonperturbatively $O(a)$-improved Wilson quark action, and make measurements for two values of hopping parameters sandwiching the strange quark mass determined by $m_{\phi}$.

We employ two finite spatial momenta of $|\vec{p}|=2 \pi / L, \sqrt{2} \cdot 2 \pi / L$ to extract the kinetic masses. Errors are estimated by the single elimination jackknife procedure for all measured quantities.

\section{Nonperturbative determination of $v$}

If all the improvement parameters in the RHQ action are nonperturbatively determined, the remaining systematic errors are $f_{2}\left(m_{Q} a\right)\left(a \Lambda_{\mathrm{QCD}}\right)^{2}$ where $f_{2}$ is an analytic function around $m_{Q} a=$ $0[1]$. The RHQ(PT) action, however, is left with the systematic errors of $O\left(\alpha_{s}^{2}\right) \sim 5 \%$ originating from $v \sum_{i} \bar{q} \gamma_{i} D_{i} q$, which is responsible for the $M^{\text {pole }}-M^{\text {kin }}$ difference and relevant for hyperfine splitting as shown below. In the case of the RHQ(NP) action, where $v$ is nonperturbatively adjusted to satisfy $M_{h h}^{\text {pole }}=M_{h h}^{\mathrm{kin}}$, the leading systematic error except for $f_{2}\left(m_{Q} a\right)\left(a \Lambda_{\mathrm{QCD}}\right)^{2}$ is $O\left(\alpha_{s}^{2} a \Lambda_{\mathrm{QCD}}\right) \sim 1 \%$ from the Wilson and the clover terms, which is negligibly small compared to the statistical errors.

In Fig.1 (a) we plot the effective speed of light $c_{\text {eff }}$ for the heavy-heavy pseudoscalar meson determined from the dispersion relation $E^{2}=m_{\text {pole }}^{2}+c_{\text {eff }}^{2}|\vec{p}|^{2}$. It is clear that the nonperturbative tuning of $v$ is successfully implemented. Figure 1(b) shows $c_{\text {eff }}$ for the heavy-light case. An important observation is that $c_{\text {eff }}$ is automatically tuned to be unity, once $v$ is adjusted by the heavy-heavy spectrum. This is expected from our formulation. 

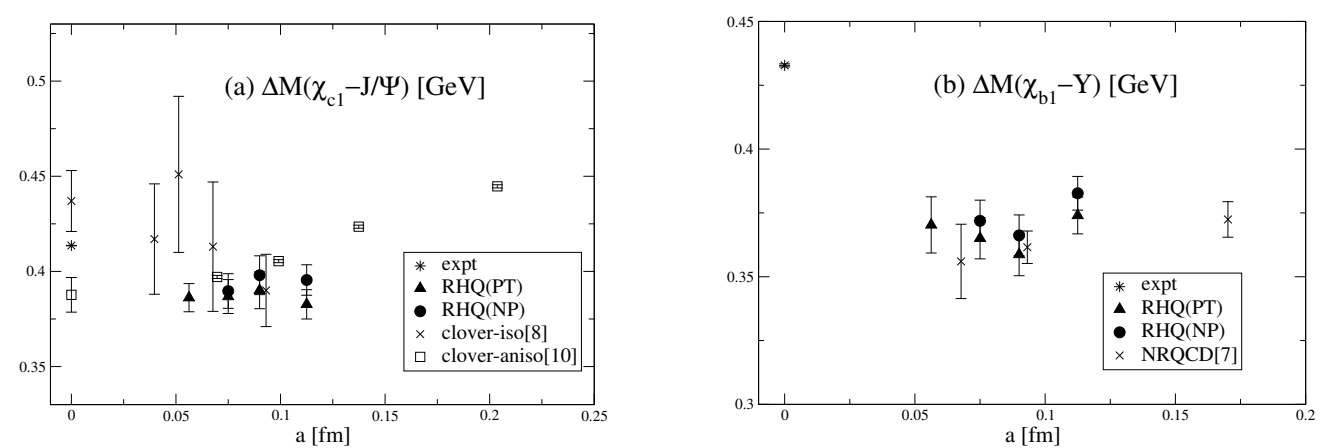

Figure 2: Orbital excitation for (a) charmonium and (b) bottomonium systems.

\section{Scaling properties for various physical quantities}

We focus on the scaling properties for charmonium and bottomonium spectra, heavy-light pseudoscalar decay constants and charm and bottom quark masses. Our results are compared to previous works with NRQCD[7] and the clover quark action on isotropic [8, 9] and anisotropic [10] lattices, whose lattice spacings are converted to those determined by $r_{0}=0.5 \mathrm{fm}$ with the aid of Ref.[11] if necessary.

Let us first present the results on quarkonium spectra. Figure 2 shows the cutoff dependence of orbital excitation: the mass difference between the ${ }^{3} P_{1}$ and ${ }^{3} S_{1}$ states. We observe good scaling behavior both for the charmonium and the bottomonium. It seems that the difference between RHQ(PT) and RHQ(NP) causes little effects on this quantity. For the bottomonium our results are consistent with those with NRQCD even at finite lattice spacing. In Fig.3 we plot the hyperfine splitting as a function of lattice spacing, which is measured with both the pole mass and the kinetic mass for RHQ(PT) and the pole mass for RHQ(NP). For the charmonium our results with RHQ(PT) and RHQ(NP) seem to converge toward the continuum values of the clover results on the isotropic and anisotropic lattices as the lattice spacing decreases. It is clear that the RHQ(NP) results show smaller scaling violation effects than the RHQ(PT) results. On the other hand, we observe rather large scaling violation effects in the bottomonium case. Although the experimental value of $\Delta M(\Upsilon$ $\eta_{b}$ ) is not known, our results appear converging around $30 \mathrm{MeV}$ toward the continuum limit. We
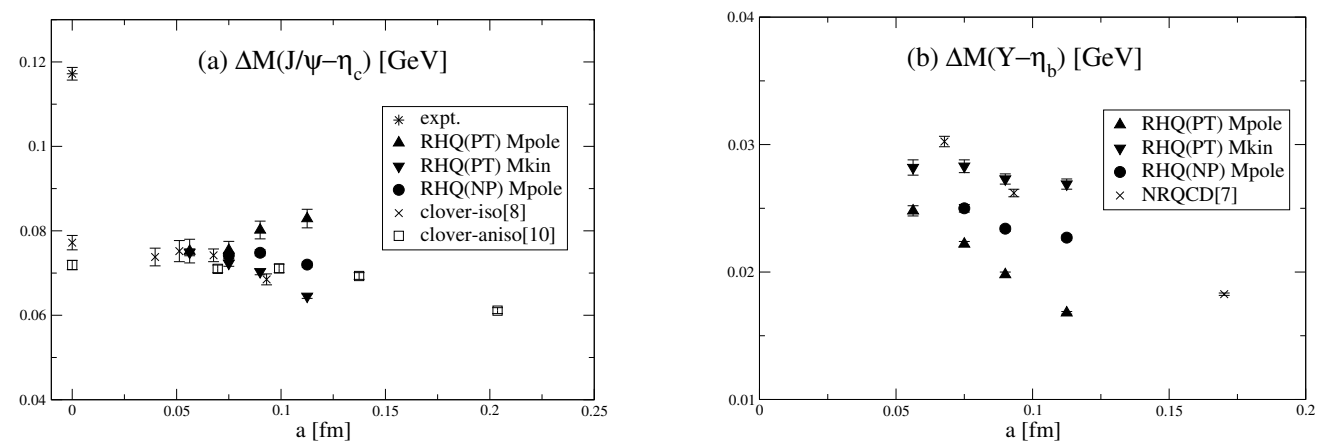

Figure 3: Hyperfine splitting for (a) charmonium and (b) bottomonium systems. 

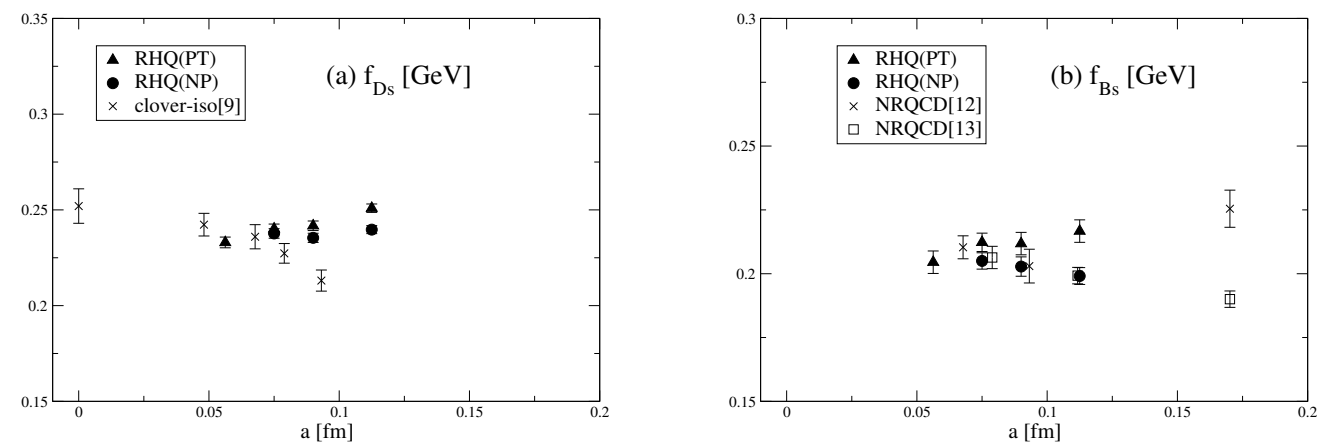

Figure 4: Decay constant for (a) $D_{s}$ and (b) $B_{s}$.

find that the NRQCD results show a stronger cutoff dependence than ours, and it is unclear how large the systematic errors are.

The heavy-light pseudoscalar decay constant is calculated using $\left\langle 0\left|A_{4}\right| P S\right\rangle=i f_{P S} m_{P S}$ with $m_{P S}$ the meson pole mass. We adopt the perturbative values for the renormalization factor and the improvement coefficients of the axial vector current[3]. The results for $f_{D_{s}}$ and $f_{B_{s}}$ in Fig 4 show good scaling behavior. The difference between RHQ(PT) and RHQ(NP) is rather small both for $f_{D_{s}}$ and $f_{B_{s}}$. Our result for $f_{D_{s}}$ shows milder cutoff effects than the clover result[9] as expected, and for $f_{B_{s}}$ good consistency is observed between our results and the NRQCD results [12, 13] at finite lattice spacings.

Let us turn to the charm and bottom quark masses in the $\overline{\mathrm{MS}}$ scheme determined from the heavy-heavy and heavy-light axial Ward identities. For the heavy-light case we employ

$$
\begin{aligned}
m_{D_{s}}\left\langle 0\left|A_{4}\right| D_{s}\right\rangle & =\left(m_{c}+m_{s}\right)\left\langle 0|P| D_{s}\right\rangle & & m_{D_{s}} \text { input }, \\
m_{B_{s}}\left\langle 0\left|A_{4}\right| B_{s}\right\rangle & =\left(m_{b}+m_{s}\right)\left\langle 0|P| B_{s}\right\rangle & & m_{B_{s}} \text { input, }
\end{aligned}
$$

where zero spatial momentum is imposed on the $D_{s}$ and $B_{s}$ states. The strange quark mass $m_{s}$ is determined by $m_{\phi}$. The renormalization factors and the improvement coefficients for $A_{4}$ and $P$ are perturbatively evaluated. We can also determine the charm and bottom quark masses from the heavy-heavy axial Ward identities:

$$
\begin{array}{ll}
m_{\eta_{c}}\left\langle 0\left|A_{4}\right| \eta_{c}\right\rangle=2 m_{c}\left\langle 0|P| \eta_{c}\right\rangle & m_{J / \Psi} \text { input }, \\
m_{\eta_{b}}\left\langle 0\left|A_{4}\right| \eta_{b}\right\rangle=2 m_{b}\left\langle 0|P| \eta_{b}\right\rangle & m_{\Upsilon} \text { input. }
\end{array}
$$

We adopt the vector meson masses as input for the heavy-heavy case, since the $\eta_{b}$ state of the bottomonium is not confirmed experimentally. In Fig. 5 we plot the RHQ(NP) results of $m_{c}^{\overline{\mathrm{MS}}}(\mu=$ $\left.m_{c}^{\overline{\mathrm{MS}}}\right)$ and $m_{b}^{\overline{\mathrm{MS}}}\left(\mu=m_{b}^{\overline{\mathrm{MS}}}\right)$. We find that the scaling violation effects are tiny for $m_{c}^{\overline{\mathrm{MS}}}\left(\mu=m_{c}^{\overline{\mathrm{MS}}}\right)$ both in the heavy-light and heavy-heavy cases, while they are sizable for $m_{b}^{\overline{\mathrm{MS}}}\left(\mu=m_{b}^{\overline{\mathrm{MS}}}\right)$.

Except for the $f_{2}\left(m_{Q} a\right)\left(a \Lambda_{\mathrm{QCD}}\right)^{2}$ contributions, the leading systematic errors in the calculation of $f_{D_{s}, B_{s}}$ and $m_{c, b}$ are $O\left(\alpha_{s}^{2}\right)$ coming from higher order effects in the renormalization factors. We plan to remove this systematic error by determining the renormalization factors nonperturbatively. Once this is achieved, the remaining systematic errors should be $O\left(\alpha_{s}^{2} a \Lambda_{\mathrm{QCD}}\right)$. 

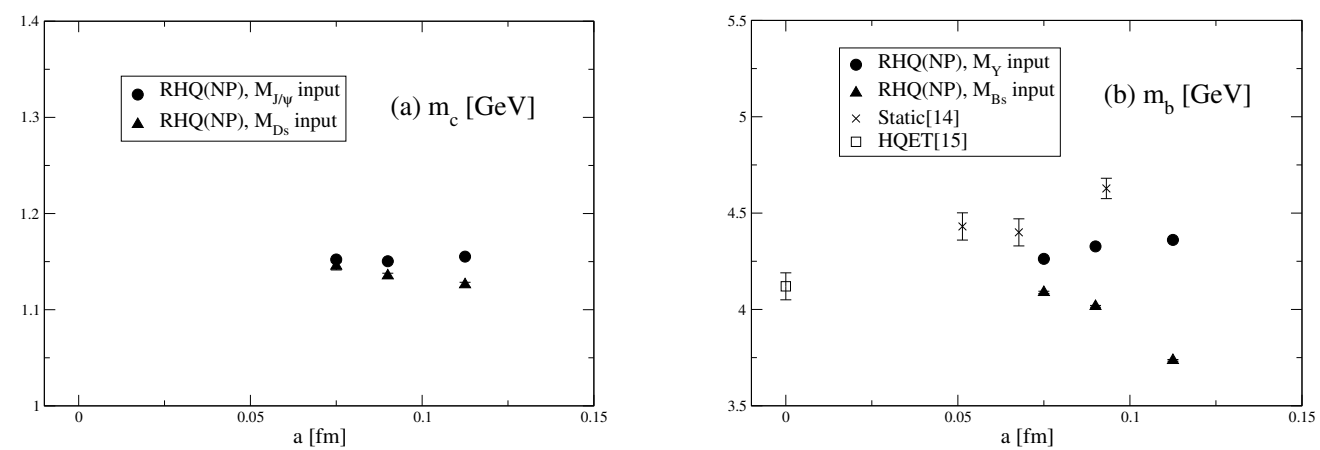

Figure 5: (a) Charm quark mass renormalized at the scale of its own mass in $\overline{\mathrm{MS}}$ scheme. (b) for bottom.

\section{Conclusions and Outlook}

The RHQ action shows good scaling behaviors both for the charm and bottom systems. Especially, once the parameter $v$ is nonperturbatively determined, the scaling properties are further improved. As a next step we are now working on a perturbative determination of the renormalization factors and the improvement coefficients for the four-fermi operators, which is relevant for the calculation of $B_{B}$. We also start to repeat the calculation on $2+1$ flavor gauge configurations generated by the CP-PACS/JLQCD Collaboration[16].

This work is supported in part by Grants-in-Aid of the Ministry of Education (No. 13135204, 13640260, 14046202, 14740173, 15204015, 15540251, 15540279, 15740134, 15740165, 16028201, 16540228, 17340066).

\section{References}

[1] S. Aoki, Y. Kuramashi and S. Tominaga, Prog. Theor. Phys. 109 (2003) 383.

[2] S. Aoki, Y. Kayaba and Y. Kuramashi, Nucl. Phys. B697 (2004) 271.

[3] S. Aoki, Y. Kayaba and Y. Kuramashi, Nucl. Phys. B689 (2004) 127.

[4] N. Yamada, S. Aoki and Y. Kuramashi, Nucl. Phys. B713 (2005) 407.

[5] Y. Iwasaki, preprint, UTHEP-118 (Dec. 1983), unpublished.

[6] CP-PACS Collaboration, S. Takeda et al., Phys. Rev. D70 (2004) 074510.

[7] C. T. H. Davies et al., Phys. Rev. D58 (1998) 054505.

[8] QCD-TARO Collaboration, S. Choe et al., JHEP 0308 (2003) 022.

[9] A. Jüttner and J. Rolf, Phys. Lett. B560 (2003) 59.

[10] CP-PACS Collaboration, M. Okamoto et al., Phys. Rev. D65 (2002) 094508.

[11] S. Necco and R. Sommer, Nucl. Phys. B622 (2002) 328.

[12] S. Collins et al., Phys. Rev. D63 (2001) 034505.

[13] JLQCD Collaboration, K-I. Ishikawa et al., Phys. Rev. D61 (2000) 074501.

[14] G. Martinelli and C. T. Sachrajda, Nucl. Phys. B559 (1999) 429.

[15] R. Sommer, hep-ph/0412209.

[16] CP-PACS Collaboration, T. Ishikawa et al., these proceedings. 